\title{
Proceso de recaudación y uso de los impuestos obtenidos en el mercado Alfredo Lazo en el año 2016 ${ }^{1}$
}

\author{
Freydel Magaly Rodríguez Salgado² \\ Mauren Karina Pérez Castellón ${ }^{3}$ \\ Freydell María Moreno Tercero ${ }^{4}$ \\ Beverly Castillo Herrera ${ }^{5}$
}

\section{RESUMEN}

El presente trabajo se refiere al Proceso de Recaudación y Uso de los Impuestos obtenidos en el Mercado Alfredo Lazo realizado por la Alcaldía de Estelí durante el año 2016. Por lo tanto, el objetivo general es analizar el proceso de la obtención y utilización de los fondos para el diseño de una estrategia que le permita a la Alcaldía la mejora del proceso de recaudación en el Mercado Alfredo Lazo del Municipio de Estelí. El enfoque de la investigación es cuali-cuantitativa con un universo de 154 tramos actualmente activos. Se aplicaron entrevistas, encuestas y guía de observación para brindar veracidad a la información brindada en el documento. Los ejes teóricos de este estudio son: Municipio, Competencia Municipal y Recaudación y las principales estrategias diseñadas y propuestas son: Promover el pago de impuestos de los comerciantes, Dar a conocer a los comerciantes del mercado en que se utilizan los impuestos recaudados, Registrar en el sistema a todos los comerciantes del mercado y garantizar un buen trabajo con una atención más eficaz por parte de los recaudadores.

Palabras clave: Recaudación, impuestos, municipio, alcaldía, mercado, Estelí.

Recibido: 12 de diciembre de 2016

Aceptado: 16 de febrero de 2017

1 Este artículo se basa en la investigación titulada Proceso de recaudación y uso de los impuestos obtenidos en el Mercado Alfredo Lazo en el año 2016. Para obtener el título de Licenciado en Administración de Empresas de la UNAN-Managua, FAREM-Estelí.

2 UNAN-Managua FAREM-Estelí, Correo electrónico: freydellrodriguez@yahoo.com

3 UNAN-Managua FAREM-Estelí, Correo electrónico: mkperez@hotmail.com

4 UNAN-Managua FAREM-Estelí, Correo electrónico: freydelita@yahoo.es

5 Doctora en Ciencias Sociales por la Universidad del Zulia, Venezuela. Maestría en Ciencias Sociales por la Universidad de Guadalajara, México. Docente titular de la UNAN-Managua, FAREM-Estelí, Correo electrónico: beverly.castillo@yahoo.com 


\title{
Process of collection and use of the taxes obtained in the market Alfredo Lazo in the year 2016
}

\begin{abstract}
The present investigative work refers to the process of collection and use of taxes obtained at Alfredo Lazo marketplace done by the city hall of Estelí during the year 2016. Therefore, the general objective is to analyze the process of obtaining and use of funds for a strategy design that allows the city hall improves the process of tax collection at Alfredo Lazo marketplace of the municipality of Estelí. The focus of the research is mixed with a universe of 154 sections currently active. We applied interviews, surveys and guide of observation to provide veracity to the information provided in this document. The theoretical axes of this study are: municipality, municipality competence, taxes collection. Thus, the main strategies designed and proposed are: promote paying taxes from traders, to inform traders of the marketplace the proper use of the taxes collected register in the city hall system all traders of the marketplace and guarantee a quality work with more effective attention from tax collectors.
\end{abstract}

Keywords: Collection, taxes, municipality, city hall, marketplace, Estelí 


\section{INTRODUCCIÓN}

En la zona sur de la ciudad de Estelí se encuentra ubicado el Mercado Alfredo Lazo, ahí se comercializan productos perecederos, granos básicos, marisquería, carnes, productos lácteos, ropa, calzado, entre otros. La Alcaldía Municipal de Estelí cuenta con un área destinada a la recaudación de impuestos, esta área es de vital importancia para el desarrollo del municipio, porque las recaudaciones permiten llevar a cabo obras de progreso y desarrollo en el mercado municipal. En la actualidad este mercado ha venido presentando una problemática en la recaudación porque los comerciantes de manera irresponsable no pagan en tiempo y forma sus impuestos, ocasionando el retraso de inversiones y mejoras.

El desorden organizacional en el Mercado Alfredo Lazo ha venido provocando que muchos comerciantes no estén legalmente inscritos ante la autoridad correspondiente y no paguen todos los impuestos. Esta situación es un problema de mala aplicación de planes y controles debido a la ausencia de una estrategia organizativa, ya que no existe un sindicato de comerciantes organizados con capacidades y actitudes que se requieren para buscar una solución ante una eventualidad problemática que se dé dentro del mercado. Se espera un impacto positivo, observar la visión de los comerciantes respecto a la manera de administrar los recursos de inversión con los que cuenta el mercado, así como el ordenamiento en lo antes mencionado y la contribución que ellos puedan hacer para mejorar su ambiente laboral y de este modo se mejoran las condiciones de compra de los consumidores y reducir situaciones de riesgo físico, laboral y sanitario, así como un mejor control de las autoridades respecto a la recaudación de impuestos.

Esta investigación permite recoger información pertinente a lo que constituyen los impuestos Municipales, los fundamentos legales en que se basan y los mecanismos de recaudación que puede aplicar la
Alcaldía, como ente responsable de la administración municipal, requiriendo así la aplicación de teorías y principios básicos relacionados con la materia tributaria, específicamente sobre los Impuestos Municipales del Mercado Alfredo Lazo, para que a través de un adecuado método estos resultados permitirán conocer como es el sistema de recaudación de los impuestos del Mercado. En lo Metodológico este trabajo se puede considerar una guía para la orientación de posteriores investigaciones, el estudio ayuda a que el proceso de recaudación de impuestos pueda visualizar cuales son las fortalezas, debilidades, oportunidades y amenazas del proceso de recaudación llevado actualmente para así proponer estrategias que le permitan mejorar la captación tributaria a dicho ente gubernamental. Los resultados de esta investigación constituyen un aporte al desarrollo, fortalecimiento y crecimiento de la administración tributaria del municipio de Estelí de tal manera que cuente con elementos básicos para analizar el rendimiento de los impuestos que administra, es decir, que el estudio sirva como instrumento para los funcionarios. Para así lograr una eficiente recaudación de los impuestos del Mercado y hacer de ella una institución capaz de generar recursos propios para satisfacer las necesidades del mercado municipal de Estelí.

Como objetivo principal se planteó, analizar el proceso de la obtención y utilización de los fondos para el diseño de una estrategia que le permita a la Alcaldía la mejora del proceso de recaudación en el mercado Alfredo Lazo en el municipio de Estelí durante el periodo 2016.

Entre los ejes teóricos de la investigación son:

Los municipios son entidades básicas de la organización territorial del estado los cuales gestionan con autonomía el interés propio de las correspondientes colectividades teniendo personalidad jurídica, plena capacidad para el cumplimiento de sus fines en el cual su gobierno y administración corresponde a los 
respectivos ayuntamientos integrados por alcaldes y concejales. (Collado, 2006).

Los gobiernos municipales tienen competencia en todas las materias que incidan en el desarrollo socioeconómico y en la conservación del ambiente y los recursos naturales de su circunscripción territorial. (Rocha, 2002).

El concepto de recaudación fiscal es aquel que se aplica al acto que realiza un organismo, normalmente el Estado o el Gobierno, con el objetivo de juntar capital para poder invertirlo y usarlo en diferentes actividades propias de su carácter. La recaudación fiscal es hoy en día un elemento central para todos los gobiernos ya que esos no son más que los fondos que podrá manejar el gobierno y que deberá asignar a diferentes espacios tales como administración pública, educación, salud, medio ambiente, trabajo, comunicación. (Franco, 2016).

\section{MATERIALES Y MÉTODOS}

Para llevar a cabo el estudio de la recaudación y uso de los impuestos obtenidos por parte del mercado Alfredo Lazo de la ciudad de Estelí en el año 20016, se utilizaron fuentes primarias de carácter cuantitativo cuyo instrumento fueron encuestas aplicadas a los comerciantes del Mercado Alfredo Lazo donde se extrajo información acerca de la recaudación y uso de los Impuestos obtenidos.

El universo de este estudio está compuesto por una población de 154 tramos activos y legalmente constituidos en la Alcaldía Municipal, siendo administrado por los comerciantes propietarios, con la realización de los cálculos correspondientes y teniendo un margen de error del 5\% y un nivel de confianza del 95\%, se obtuvo un tamaño de la muestra de 110 comerciantes.
Así mismo se aplicó entrevistas dirigidas a la Lic. Jackeline Torres (Responsable del Área de Recaudación de la Alcaldía Municipal de Estelí) y al señor Arturo Molina (Intendente del Mercado Alfredo Lazo del municipio de Estelí). También se hizo una revisión bibliográfica de diferentes libros y trabajos de curso para enriquecer y dar veracidad a la información obtenida durante esta investigación.

Para la elaboración de instrumentos de recolección de datos se tomó en cuenta el contenido del cuadro de operacionalización de los objetivos específicos, para la realización de guía de entrevistas al personal encargado de cada una de las diferentes áreas abordadas en nuestra investigación y encuestas dirigidas a los comerciantes, para la manipulación de los resultados de las encuestas se utilizó el programa SPSS, también se aplicó una guía de observación para abordar la problemática del mercado Alfredo Lazo. Todos los instrumentos fueron revisados para proceder a la aplicación de los mismos.

\section{RESULTADOS}

\section{Caracterización de la Alcaldía de Estelí ${ }^{1}$}

Las alcaldías son instituciones que se encuentran encargadas de la administración de los bienes y recursos con los que cuenta la municipalidad por funcionarios que representan a la población y que fueron elegidas de manera democrática, por lo que sus responsabilidades son de suma importancia ${ }^{2}$.

La Alcaldía de Estelí está compuesta por 400 personas que laboran en las instalaciones y en otros edificios delegados a su autoridad, operando de modo que se le pueda dar respuesta a todas las necesidades que la población demande de manera rápida, con mucha eficiencia y eficacia en pro de los intereses del pueblo.

1 Alcaldía de Estelí. Entrevista. Raúdez Rafael. Secretario del Consejo Municipal. Consulta: 04/06/2016.

2 Ley de Municipios. Ley N 40 Capitulo III. Artículos 17, 18, 19.

Publicado en el Gaceta N 155 de 17 de Agosto de 1998. 


\section{Estructura organizativa de la Alcaldía de Estelí}

El gobierno y la administración municipal cumplirán

e impulsarán el cumplimiento de la Constitución Política de la Republica de Nicaragua, promoverán

los intereses del pueblo y defenderán sus conquistas sociales y políticas. El gobierno y la administración de los municipios corresponden a un consejo municipal, el cual tiene carácter deliberante, normativo y administrativo. El consejo estará presidido por un Alcalde elegido de su seno. El consejo municipal será elegido por el pueblo, mediante el sufragio universal, igual, directo, libre y secreto de acuerdo a la ley electoral. El gobierno de los municipios gozara de autonomía, sin detrimento de las facultades del gobierno central.

Según los niveles jerárquicos la máxima autoridad es el Consejo Municipal, luego está la dirección superior conformada por el Alcalde y Vicealcaldesa, que junto con el Consejo Municipal aprueba los proyectos, presupuesto o inversiones que realiza la municipalidad,

\section{Organigrama de la Alcaldía de Estelí ${ }^{4}$}

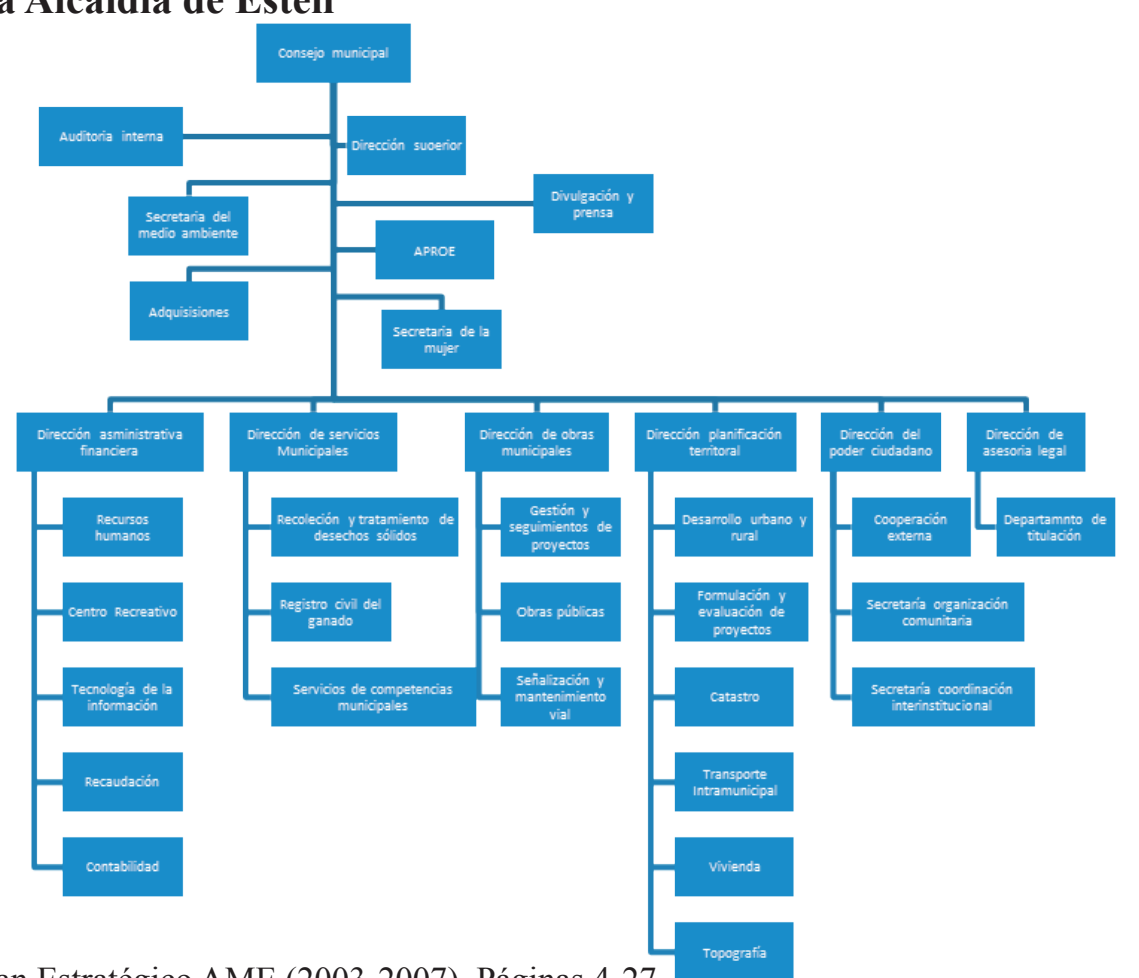

para que sean de primera necesidad y estén dentro de los límites establecidos.

\section{Misión y Visión de la Alcaldía de Estelín}

\section{Misión}

"Mejorar la habilidad, Gobernabilidad y Calidad de vida de la población en el municipio de Estelí a través de una gestión administrativa más efectiva y transparente de sus recursos humanos financieros y materiales basadas en la participación ciudadana como eje fundamental de desarrollo, la preservación del medio ambiente, enfoque de género y generacional en la promoción de respeto a los derechos humanos."

\section{Visión}

"Un Estelí como centro económico socio educativo cultural y ambiental del norte de Nicaragua que promueva el desarrollo humano con enfoque de género y generacional y una calidad de vida creciente con base en la promoción de los derechos humanos, la participación y el compromiso de la sociedad civil, la inversión del sector público y privado para un desarrollo local sostenible."

3 Alcaldía de Estelí. Plan Estratégico AME (2003-2007). Páginas 4-27

4 Lic. Marta Azucena Fuente Rocha. Directora Administrativa Financiera. Manual de Funciones de la Alcaldía de Estelí. 30 de agosto 2005 . 


\section{Proceso de Administración de Ingresos del área de Recaudación de la Alcaldía Municipal de Estelín}

La función del área de recaudación de impuestos de la Alcaldía Municipal de Estelí, es de percibir efectivamente el pago de todas las deudas tributarias de contribuyentes hacer que el deudor tributario cumpla con sus obligaciones por la vía de pago, actualizando a la vez el registro de su cuenta corriente. La base legal se determina sobre el monto gravable del impuesto, el ingreso percibido por el contribuyente en donde se determina una cuota o cantidad de dinero, una tasa porcentual. Los ingresos tributarios pueden proceder de impuestos municipales, tasas y contribuciones especiales, los que serán regulados por la ley de la materia. El consejo municipal no podrá acordar exenciones, exoneraciones o rebajas de impuestos,

tasas o contribuciones especiales, salvo en los casos previstos en la legislación tributaria municipal y de acuerdo a las formalidades establecidas en la misma. No se podrán realizar egresos superiores a los

consignados en el presupuesto municipal ni efectuar egresos en conceptos no presupuestados sin la previa reforma al mismo por el Consejo Municipal respectivo que amplié, dote o trasladé el crédito presupuestario correspondiente.

\section{Beneficios e Inversiones de la Recaudación de Impuestos}

Como parte de la Recaudación de Impuestos el Municipio de Estelí se beneficia con diferentes tipos de proyectos:

- Proyectos Educativos: Construcción de escuelas en zonas urbanas y rurales, capacitación de los docentes de los centros educativos, servicios de

5 Arq. García A. Leonardo, Mercado Municipal, Manuales Elementales de Servicios Municipales 2. agosto 2003. Edición Nina Lucia Monje. Reformar e Incorporaciones a la Ley $\mathrm{N}^{\circ} .40$, “Ley de Municipios” Pag. 85, Arto. 49-51. alimentación en centro escolares de educación primaria, compra de útiles escolares y utensilios de limpieza, creación de una biblioteca municipal, reparación de infraestructura escolar, equipamiento tecnológico de centros de educación secundaria, financiamiento de becas escolares a universitarios.

- Proyectos Sociales: Encunetado y revestimiento de calles de los diferentes barrios del Municipio de Estelí, construcción y reparación de puentes, saneamiento de aguas potables, instalación de tuberías de aguas negras, alumbrado público en las zonas rurales, señalización vial de las calles y carreteras, construcción de centros de salud, proyectos de urbanización y construcción de viviendas, limpiezas de causes, tren de aseo de los diferentes barrios.

- Proyectos de Cultura y Deporte: Financiamiento de becas deportivas, uniformes para equipos, accesorios deportivos, mantenimiento de estadios y campos deportivos, proyección social de talentos deportivos.

- Mejoras en la infraestructura del Mercado Alfredo Lazo, con el apoyo de la Procuraduría General de la Republica y Fondos del Instituto Austriaco Norte-Sur.

\section{Servicios municipales de la Alcaldía de Estelí}

El área de Servicios Municipales es la encargada de garantizar a la población de manera permanente, regular y continua sin fines de lucro los servicios que la legislación vigente atribuyen al municipio, estos servicios pueden prestarse en forma directa por la municipalidad o en forma indirecta con el fin de asegurarlos con eficiencia, continuidad y eficacia. En esta área se encuentra la unidad del Mercado Alfredo Lazo.

\section{Función de la Dirección de Servicios Municipales}

La administración del mercado Alfredo Lazo está a cargo de la Alcaldía Municipal, quien delega 
responsabilidades a la dirección de Servicios Municipales dirigida por el Ing. Carlos Pino, y este establece un intendente siendo el actual el Sr. Arturo Molina, quien es el encargado de ejecutar los planes que se elaboran a favor del mercado. Las actividades que el intendente ejecuta se limitan hacer operativas, no participa en la creación de planes u otras acciones, las autoridades de la alcaldía son las que llevan a cabo estas funciones, siendo así una consecuencia de deficiencia administrativa en el mercado. No existe un manual de funciones y procedimientos que establezcan las actividades que desempeñan los encargados del mercado.

Para determinar los criterios que se deben evaluar en el proceso administrativo actual es importante reconocer los tipos de control que la intendencia aplica en el mercado:

\section{Control de Personal que labora para el} funcionamiento del mercado: El fin es de controlar los egresos por gastos administrativos, como el pago de salarios mediante el uso de planillas.

Control Financiero: El intendente se encarga de la manipulación de los recursos económicos destinados al mercado según lo estipulado en el presupuesto, debe de presentar informes mensuales a las autoridades de la Alcaldía de Estelí, en el cual se detalla la justificación de cada gasto realizado, las facturas y recibos que lo soportan de modo que sean equivalentes los ingresos y egresos de las unidades monetarias y las unidades físicas en el mismo.

\section{Control de cobro de las tasas y derechos que deben} pagar todos los comerciantes: Para lo cual existe una coordinación muy estrecha de los cobradores quienes los determinan las autoridades de la Alcaldía Municipal, proveniente de departamento administrativo o de recaudación, por lo que la intendencia se limita a controlar el proceso, pero no lo ejecuta completamente, es decir que no tiene contacto con el dinero que se recauda de esas tasas, sino que se lleva directamente a la Alcaldía Municipal. El intendente vigila que exista un adecuado trato entre los cobradores y comerciantes, pero en dadas ocasiones se han visto enfrentamiento entre ellos por desacuerdos sobre la cuota.

Control de Inventario: Se aplica directamente por el intendente, aunque no existan grandes cantidades o reservas de material, útiles e instrumentos de oficina ya que actualmente solo se cuenta con dos escritorios de madera y cuatro sillas de plástico.

Control de Inversión: Como todas las funciones de un administrador, el control sobre el estudio de las futuras inversiones está a su cargo, en este caso debería ser el Intendente del mercado pues es la persona que conoce de cerca cómo se desarrollan los procesos y cuáles son sus prioridades en base a sus necesidades, pero el control sobre la inversión lo realizan las autoridades municipales quienes determinan un monto estimado. La intendencia únicamente realiza un plan de inversión según las necesidades básicas, pero son las autoridades de la Alcaldía quienes determinan su aprobación y modificación.

\section{Control de los Comerciantes Legalmente} Establecidos: Se da mediante el registro de estos ante la municipalidad, verificando cambios en los datos personales para su adecuada actualización, verificación de su situación real de ventas y control del espacio que utilizan para realizar sus ventas, con la ayuda de un mapa del mercado que describe el espacio correspondiente a cada tramo.

\section{Antecedentes del Mercado Alfredo Lazo del Municipio de Estelí}

Las instalaciones actuales donde se encuentra ubicado el mercado Alfredo Lazo, fueron construidas en 1980, con el propósito de establecer en ellas una terminal de buses con pequeños establecimientos de ventas populares de comidas rápidas, cafetines y otros 
servicios para las personas que utilizaban dicho local, con la población aproximada de 45,000 habitantes.

Por la creciente demanda del servicio de transporte y las necesidades de los usuarios, surgen pequeños puestos perecederos, frutas, carnes y productos varios con dimensiones de 3 mts2 cada uno, definiendo así las autoridades municipales 7 puestos de productos alimenticios, 9 puestos de comida rápida y 8 módulos de ropa y calzado, operando regularmente durante 3 años.

En 1983 no estaba formalmente establecida la Cooperativa de Transporte del Norte (COTRAN R.L), por lo cual al presentarse en ese momento conflictos armados, la infraestructura es utilizada como refugio para pobladores de la zona de Miraflor que habían sido afectados por los ataques de la contra revolución en el periodo de la guerra, es entonces cuando surgen los comedores y cafetines para atender las necesidades de estas personas, permaneciendo así hasta la actualidad sin tomar en cuenta las medidas higiénicas exigidas por el MINSA para la constitución de negocios de este tipo.

Al concluir los enfrentamientos armados, la situación socioeconómica de la ciudad se estabiliza y a partir de ello el mercado empieza a tomar formas incrementando la comercialización de ropa, granos básicos y productos perecederos, venta y comida y carnes, así como otros servicios que ahí se ofrecen para la población, lo cual provoco un exceso de comerciantes y vendedores ambulantes, obteniendo así una terminal desordenada y con poca capacidad de transporte, carga y descarga.

Se construye la terminal de buses Norte y Sur en 1998, para el traslado voluntario de los transportistas a falta de comisiones de la terminal anterior, una vez ubicada a los transportistas y desalojada las instalaciones anteriores, los comerciantes y pobladores vecinos toman por cuenta propia y sin autorización legal el terreno baldío durante la noche tramos asentando sus y puestos de venta de diferentes rubros. Luego estos se dieron su espacio a personas con capacidad de venta por montos elevados $(\$ 4,000.00$ dólares $)$ aproximadamente, el mismo costo de una casa particular en ese momento.

A finales del mismo año Nicaragua atraviesa una catástrofe natural de gran magnitud (Huracán Mitch) por lo que las autoridades fijaron su vista en asuntos de prioridad y desatendieron la problemática de la antigua terminal de buses, propiciando a que los comerciantes tomaran el espacio que ellos desearan, dando origen a puestos de ventas de diferentes dimensiones y con propietarios repetitivos.

\section{Proceso de toma de decisiones}

En el caso de decisiones determinantes que afecten no solo al personal que está a cargo de la intendencia, sino a los comerciantes que laboran en el mercado, se procede a realizar reuniones en las cuales participa el Intendente, El Director de Servicios Municipales, Representantes de las Redes Empresariales y Miembros de la Comisión del Poder Ciudadano, para lo cual se llega a un consejo en conformidad con todas las partes involucradas. Hasta el momento se ha aplicado este tipo de reuniones por la resistencia de los comerciantes al no pago de impuestos, al ordenamiento de comerciantes ilegales y para la futura ejecución de proyectos en el Mercado.

En el caso de las decisiones de menor impacto están a cargo del Intendente, tal es el caso de arreglos de pago, prorrogas de estadía por disminución de ventas, aplicación de sanciones, mediación entre los comerciantes conflictivos, etc.

La dirección del mercado estábajo control del Intendente quien toma las decisiones en base a las necesidades y en beneficio de los comerciantes y consumidores. No se cuenta con el apoyo de las autoridades superiores, ya que solo conocen los montos que genera el mercado, no así las necesidades. 
El Intendente dirige la organización de los tramos y arrendatarios por tipo de actividad, según una planificación establecida. Está a cargo del Intendente dirigir la limpieza de las instalaciones del mercado, a fin de que se realice en tiempo y forma en coordinación con la Dirección de Servicios Municipales, con la periodicidad requerida y establecida en horarios adecuados, depositando los desechos en el contenedor respectivo.

\section{Administración del Mercado Alfredo Lazo}

El mercado Alfredo Lazo es un área de la Alcaldía Municipal, estos funcionan en conjunto, mediante una comunicación directa con el área de recaudación. Cuenta con un recaudador de impuestos, el cual es puesto por la oficina de Recaudación.

El encargado del Mercado solo recibe la información precisa de cómo va la recaudación, los comerciantes en mora, para darle seguimiento a las situaciones que se dan en el Mercado. La administración del Mercado no manipula el dinero que se obtiene de las recaudaciones, lo maneja directamente la oficina de Recaudación de la Alcaldía de Estelí.

La administración del Mercado si maneja los datos estadísticos de la información y se le da seguimiento, control, porque son los encargados de realizar los arreglos de pagos, los cobros con los comerciantes que están en mora, y es quienes extienden las debidas notificaciones a los comerciantes, siempre trabajando en conjunto con el recaudador de la Alcaldía.

\section{Funciones de la Administración del Mercado Alfredo Lazo}

Como parte de las funciones que la administración del mercado debe llevar, se toma en consideración:

Los planes estratégicos y operativos: La intendencia lo aplica en conjunto con los comerciantes y cada una de las áreas involucradas. El monitoreo y seguimiento de estos planes los aplica y los ejecuta la intendencia para realizar evaluaciones de su efectividad. No existe un plan de acción para solucionar problemas de asentamiento de comerciantes ilegales.

Planes Presupuestales: La intendencia ofrece a las autoridades municipales una propuesta de inversión en base a los gastos que se han tenido en los últimos meses, priorizando aquellos recursos para asegurar las operaciones futuras del mercado, pero la aprobación de esta propuesta está en manos de la Administración de la Alcaldía. La propuesta se presenta de manera anual en el mes de noviembre antes de la Asamblea Presupuestal.

Planificación de las reuniones: Se realizan para tratar asuntos en relación a las actividades de seguridad del mercado y programar las supervisiones y controles por parte de las autoridades sanitarias (MINSA), para garantizar que los comerciantes cumplan con las debidas medidas sanitarias al momento de comercializar los productos que ofrecen, las reuniones se realizan cada tres meses en las instalaciones del mercado, dicha área no cuenta con una sala reuniones acorde para esta función.

Planificación de las Capacitaciones: La intendencia no cuenta con los recursos necesarios para llevar este proceso, por lo cual las capacitaciones se hacen una vez al año, con el fin de mejorar las condiciones de venta e incrementar los niveles de demanda y capacitar al personal que labora para el mercado.

\section{Procedimiento para instalarse en el mercado}

- El comerciante interesado en adquirir un tramo en el Mercado Alfredo Lazo debe visitar al intendente encargado para acelerar las gestiones; ya que esta persona es la que maneja que tramos están vacantes y cuál es la ubicación de cada tramo.

- Al haber disponibilidad de tramos el encargado 
del mercado le solicitara al comerciante los siguientes requisitos. Llevar dos fotografías, llenar una solicitud, llevar dos cartas de recomendación, entregar una fotocopia de cedula, presentar su record de policía.

- El comerciante posteriormente deberá dirigirse a la Alcaldía Municipal indagando información con la Lic. Melania Peralta Vicealcaldesa, quien proporcionará información y la debida aprobación para el arrendamiento del tramo, siempre y cuando el comerciante cuente con todos los requisitos correspondientes. Para la solicitud de un servicio el contribuyente o comerciante debe cumplir con los requisitos que se le impone en la inscripción.

- Si el comerciante es aprobado y cumple con los requisitos se le asigna el tramo tomando en cuenta la solvencia económica que tenga con la Alcaldía.

- Toda persona natural o jurídica que se dedique a la venta o prestación de servicio deberá solicitar la matricula del negocio anualmente en el municipio para cada una de las actividades económicamente diferenciada que en el mismo se desarrolle. La matrícula deberá efectuarse en Enero de cada año.

- Los comerciantes pagarán mensualmente un impuesto municipal del $2 \%$ sobre sus ventas.

- También deberán pagar el impuesto por arrendamiento de tramo y por el servicio de tren de aseo.

\section{Organización del Mercado Alfredo Lazo}

El uso de las instalaciones: Está a cargo del intendente que vigila que se aprovechen de manera adecuada y se resguarden, para ello se organiza con todos los comerciantes legalmente establecidos por medio de los representantes a fin de simplificar el proceso comunicativo entre todo ellos según su rubro.

La organización de los puestos de venta: La ejecuta el intendente del mercado con máxima autoridad representante de las autoridades municipales según convenga al máximo provecho del espacio y los límites del mercado. Se organizan de la siguiente manera: perecederos 1 , perecederos 2 , cafetines, comercio y servicios, granos básicos, carniceros y lácteos.

En los ingresos por canal de arriendo: La intendencia no participa en esta actividad para lo cual se limita a supervisar y se organiza con los recaudadores establecidos por la alcaldía de Estelí para realizar el proceso de conjunto.

Organización con los comerciantes: La intendencia del mercado se organiza mediante reuniones con el objetivo de conocer los problemas que más le afecten, escuchar sus quejas, demandas o sugerencias a la forma de administrar el mercado. Se realiza un determinado proceso para poder realizarla:

- Se reúnen los comerciantes, el director de servicios municipales y el Intendente del mercado.

- La solicitud de la reunión se realiza mínimo con 10 días de anticipación.

- Se realizan una vez al mes

- Se discuten asuntos del mercado y de interés para ambas partes. Entre algunas están: Solicitud de información del mercado, Seguridad de sus tramos, Enfrentamiento de comerciantes legales y ambulantes, Arreglos de pagos de los impuestos.

Limpieza de cada tramo: Los comerciantes deben de limpiar sus tramos 3 veces al día específicamente para los productos perecederos, la limpieza será supervisada por una persona que el intendente destine, lo cual revisara cada tramo legalmente inscrito. Si el propietario del tramo no cumple será sancionado con una multa de C $\$ 50.00$.

- El aseo de vías públicas, aceras y cunetas: Lo realizan las brigadas de limpieza compuestas por 9 personas quienes se desplazan por todo el mercado.

- La recolección y transporte de la basura: Es controlado por la intendencia del mercado solicitado a la Dirección de Servicios Municipales. 
- Aseguramiento de un contenedor: Cuya capacidad no es suficiente puesto que solo logra almacenar dos toneladas de basura.

- Seguridad para compradores: Actualmente no se presta la seguridad a los comerciantes, ni a consumidores; pues no se cuenta con recursos suficientes para contratar personal que brinde su servicio durante el día.

- Seguridad peatonal y vehicular: Ambos deberán tener cautela y precaución ya que no se ha logrado el descongestionamiento vehicular, evitando el tráfico de estos en las vías principales del mercado.

- Seguridad nocturna: Se garantiza a los comerciantes dueños de tramos vigilantes contratados por la intendencia.

\section{Valoración de los comerciantes del Mercado Alfredo} Lazo sobre la recaudación e inversión

Según los datos procesados de encuesta el mayor porcentaje de comerciantes con un $89 \%$ consideraron que si se han beneficiado con obras de progreso ya que la estructura del mercado ha mejorado, así como sus condiciones higiénicas y la seguridad de los mismos. Con un 63\% de los comerciantes considera que la Alcaldía representa transparencia al momento de captar el pago de sus impuestos, ya que son visitados directamente en el mercado brindándoles la información correspondiente de sus pagos. Todos los comerciantes consideran que si existen irregularidades en la recaudación de impuestos, ya que muchos comerciantes ilegales no pagan sus impuestos y la Alcaldía no implementa estrategias para reorganizar el mercado y así beneficiarlos a ellos evitando ventas fraudulentas.

En el Mercado Alfredo Lazo existen 154 comerciantes legalmente inscritos estos están distribuidos de la siguiente manera:
Tabla Nº 1: Distribución de los comerciantes del Mercado Alfredo Lazo. Universo: 154 comerciantes

\begin{tabular}{llc}
\hline $\mathbf{N}^{\circ}$ & \multicolumn{1}{c}{ Actividad } & Cantidad de tramos \\
\hline 1 & Mariscos & 1 \\
2 & Cafetín & 14 \\
3 & Miscelánea & 2 \\
4 & Pulpería & 2 \\
5 & Granos Básicos & 3 \\
6 & Barbería & 1 \\
7 & Salón de Belleza & 1 \\
8 & Venta de Plástico & 2 \\
9 & Ferretería & 1 \\
10 & Zapatería & 2 \\
11 & Carnicería & 8 \\
12 & Bar & 1 \\
13 & Comedores & 7 \\
14 & Tienda & 14 \\
15 & Verduras & 71 \\
16 & Lácteos & 8 \\
17 & Móviles & 28 \\
\hline & & 154 \\
\hline
\end{tabular}

Fuente: Alcaldía Municipal de Estelí, 2016.

Funcionamiento para el proceso de administración de ingresos del Mercado Alfredo Lazo

\section{Organización}

- El mercado Alfredo Lazo es un área de la Alcaldía Municipal, estos funcionan en conjunto, mediante una comunicación directa con el área de recaudación.

- Cuenta con un recaudador de impuestos, el cual es puesto por la oficina de Recaudación.

- El encargado del Mercado solo recibe la información precisa de cómo va la recaudación, los comerciantes en mora, para darle seguimiento a las situaciones que se dan en el Mercado.

- La administración del Mercado no manipula el dinero que se obtiene de las recaudaciones, lo maneja directamente la oficina de Recaudación de la Alcaldía de Estelí.

- La administración del Mercado si maneja los 
datos estadísticos de la información y se le da seguimiento, control, porque son los encargados de realizar los arreglos de pagos, los cobros con los comerciantes que están en mora, y es quienes extienden las debidas notificaciones a los comerciantes, siempre trabajando en conjunto con el recaudador de la Alcaldía.

\section{Análisis de fortaleza, oportunidades, debilidades y amenazas (FODA)}

Mediante el análisis de los resultados se elaboró un análisis FODA de la institución que permitirán a la Alcaldía lograr un equilibrio organizacional y reorientar o innovar sus estrategias hacia las necesidades de los comerciantes. Estas estrategias se basaron en las debilidades y las oportunidades.

\begin{tabular}{|c|c|}
\hline Fortalezas & Oportunidades \\
\hline $\begin{array}{l}\text { Aporta al desarrollo } \\
\text { Económico de la } \\
\text { ciudad. } \\
\text { Autonomía de los } \\
\text { ingresos. } \\
\text { - Área Administrativa. } \\
\text { - Cuota Fija. } \\
\text { - Afluencia de } \\
\text { consumidores } \\
\text { diariamente. } \\
\text { Precios Accesibles. } \\
\text { Calidad del nivel } \\
\text { de vida de los } \\
\text { comerciantes. } \\
\text { Son visitados } \\
\text { directamente por los } \\
\text { proveedores. } \\
\text { Reordenamiento de los } \\
\text { tramos. }\end{array}$ & $\begin{array}{ll}\text { - } & \text { Crecimiento } \\
\text { poblacional. } \\
\text { - } \\
\text { Inversiones de } \\
\text { organismos no } \\
\text { gubernamentales. } \\
\text { - } & \text { Acceso a la tecnología. } \\
\text { - } & \text { Relaciones que tiene } \\
\text { la alcaldía con el } \\
\text { gobierno central para } \\
\text { invertir en el mercado. }\end{array}$ \\
\hline
\end{tabular}

\begin{tabular}{|c|c|}
\hline Debilidades & Amenazas \\
\hline $\begin{array}{l}\text { Incumplimiento en el } \\
\text { pago de obligaciones } \\
\text { tributarias por parte de } \\
\text { los comerciantes. } \\
\text { Inadecuado sistema de } \\
\text { control administrativo. }\end{array}$ & $\begin{array}{l}\text { - } \quad \text { Comerciantes Ilegales. } \\
\text { - Nuevos competidores. }\end{array}$ \\
\hline
\end{tabular}

- Falta de divulgación en cuanto al uso de los recursos. Falta de organización entre los comerciantes.

- Espacio físico de los módulos muy reducido.

- Higiene y presentación de los productos.

- Sensibilizar a los comerciantes a cultura de pago de impuestos.

\section{Líneas Estratégicas para mejorar el proceso de recaudación de impuestos en el Mercado Alfredo Lazo}

Después de haber establecido el análisis FODA las estrategias que se proponen están basadas en la redacción de sus debilidades para mejorar el proceso de recaudación de impuestos.

\section{Estrategia 1: Instalar un Sistema de Recaudación}

Objetivo: Cumplir con el pago de las obligaciones tributarias.

\section{Actividades:}

1. Contratación de servicios técnicos para la instalación del sistema de recaudación.

2. Capacitación para el manejo del nuevo sistema al personal del área de recaudación de impuestos.

3. Elaborar una base de datos de todos los comerciantes establecidos legamente en el mercado Alfredo Lazo.

4. Actualizar la base de datos anualmente para el control de las recaudaciones.

5. Aplicar sanciones y multas a los contribuyentes por incumplimiento de las obligaciones tributarias. 


\section{Estratégica 2: Diseño de una Estrategia de Divulgación sobre la Inversión en el Mercado} Alfredo Lazo

Objetivo: Informar a los comerciantes sobre el uso de los impuestos que pagan y la inversión que se realiza en el mercado.

\section{Actividades:}

1. Activar un punto de encuentro para reuniones donde participen los comerciantes para compartir experiencias, problemas, etc.

2. Dar a conocer a los comerciantes los proyectos que se llevan a cabo con el pago de sus impuestos.

3. Desarrollar acciones de formación para facilitar la comunicación y el intercambio entre los comerciantes, intendencia y Alcaldía Municipal.

\section{Estrategia 3: Mejorar la Infraestructura del Mercado Alfredo Lazo}

Objetivo: Mejorar las condiciones de infraestructura de los módulos para brindar una mejor atención a los clientes.

\section{Actividades:}

1. Modernizar los espacios de los tramos.

2. Ordenar tramos por actividad comercial.

3. Construir un parqueo apropiado para los vehículos de los clientes y comerciantes.

\section{Estrategia 4: Creación de un área de parqueo.}

Objetivo: Proporcionar a la población un área para el parqueo vehicular.

\section{Actividades:}

1. Construir un parqueo apropiado para los conductores de vehículos y comerciantes.

2. Descongestionar el tráfico vehicular en las principales vías de acceso al mercado.

3. Garantizar la seguridad de los vehículos.

\section{CONCLUSIONES}

Al realizar esta investigación en el Mercado Alfredo Lazo de la Ciudad de Estelí, se observan las fallas existentes y el desinterés de las autoridades municipales por el funcionamiento del mismo a pesar de ser uno de los servicios con mayor demanda por parte de la población.

La situación actual es consecuencia de la deficiencia administrativa de la alta dirección y la inexistencia de un área encargada de dicha actividad en las instalaciones del Mercado.

Se logró el cumplimiento de los objetivos propuestos al analizar el proceso de Recaudación de Impuestos que aplica la Alcaldía en dicho Mercado, momento en que se comprobó la falta de planificación de actividades encaminadas al mejoramiento del Mercado, pues se limita a aplicar acciones menores e ineficientes con ausencia de objetivos y metas proyectadas al desarrollo a corto y mediano plazo. Su organización actual en cuanto a la planta física ha mejorado considerablemente con el desarrollo del proyecto de modernización, aunque existen conflictos por la permanencia de aquellos comerciantes que se niegan a trasladarse a otro lugar o a formar parte de los puestos de venta de este mercado de manera legal.

Acciones como esta deben ser dirigidas y mediadas por las autoridades municipales pues la incapacidad de las acciones tomadas por la intendencia no ha provocado ningún afecto, limitándose actualmente a mediar en asuntos leves como: solicitudes en prórrogas de pago del canon, mediar conflictos entre los comerciantes legales del mismo y gestionar las futuras capacitaciones. Se aseguran únicamente la aplicación de controles de sanidad e higiene, con bajos niveles de exigencia lo que ha ocasionado ciertos casos de productos contaminados sobre todo en los puestos de productos cárnicos. 
También se logró conocer y analizar la visión y contribución de los comerciantes respecto al sistema de dirección actual, a lo cual la mayoría desconoce los procedimientos internos que se aplican y no están de acuerdo con actividades realizadas por personas ajenas a este mercado, como es la recaudación del canon de arrendamiento, estudios presupuestables de reinversión y el control de todos los gastos que ahí se realizan durante todo el año, a lo cual solicitaron mayor flujo de información por parte de la autoridades.

Se establecen líneas estratégicas que abarcan diferentes puntos clave del Mercado, a fin de maximizar el provecho de todos estos recursos y contar con un sistema administrativo eficiente, activo y rentable para el mercado y para los comerciantes que trabajan en el mismo.

\section{BIBLIOGRAFÍA}

Collado, P. E. (2006). Legislación Administrativa. Sevilla: 21 Edición.

Franco, E. R. (2016). Procedimiento de recaudación e inspección de los tributos. UF1817.

Rocha, J. L. (2002). El apoyo municipal a las microempresas: marco jurídico y valoración de actores claves. (2. Instituto de Investigación y Desarrollo Nitlapán, Ed.) Nitlapán. 